\title{
Contents of Next Issue of Opticheskii Zhurnal (Journal of Optical Technology)
}

DOI: $10.1134 / \mathrm{S} 0030400 \mathrm{X} 11060117$

The following papers will be published in the May issue of Opticheskii Zhurnal (Journal of Optical Technology), Vol. 78, No. 5, 2011.

Physical Optics. Manifestation of Intramolecular Vibronic Interactions in the Fine-Structure Fluorescence and Fluorescence Excitation Spectra of Polyene $\delta$-Dimethylaminoketones (I.A. Vasil'eva, V.V. Kompaneets, Zh.A. Krasnaya, and Z.A. Chizhikova); Optical Properties of Metal-Dielectric One-Dimensional Diffraction Gratings (S.A. Kuznetsov, V.I. Belotelov, A.N. Kalish, A.Vengurlekar, and A.K. Zvezdin).

Computation, Design, and Manufacture of Optical Systems. The Effect of Parameters of Catadioptric Retroreflector on Characteristics of Its Field of View (A.D. Tsvetkov).

Iconics: The Science of Images. Clipping-Blurring-Rotation Technique for Reconstruction of Distorted Images (V.S. Sizikov); Object Segmentation and Tracking against Complex Background (I.V. Borisova).
Optical Instrumentation and Technology. AntennaCoupled Microbolometers (V.Yu. Zerov, V.G. Malyarov, and I.A. Khrebtov); Submersible Meter of Light Attenuation Index of Sea Water (I.M. Levin, M.A. Rodionov, and O.N. Frantsuzov); Principles of Design of Noise-Immune Midget Pulsed Laser Range Finders, Altimeters, and Sensors for On-Board and Vehicle-Borne Systems (V.N. Legkii, B.V. Galun, S.A. Litvinenko, O.V. Sankov, V.A. Shumeiko, I.Yu. Balasov, and A.O. Bashmakov); Specific Features of Design of Angular Position Sensors of Modern Geodetic Devices (V.A. Tarkov).

Optical Materials Science and Technology. Dependences of Absorption Band Intensities in High-Purity Silica Glass on Electron-Beam Fluence (A.P. Sergeev and P.B. Sergeev); Resonance Laser Spectroscopy of Ion Pairs in $\mathrm{Nd}^{3+}: \mathrm{LaF}_{3}$ Crystal (R.A. Akhmedzhanov, A.A. Bondartsev, L.A. Gushchin, and V.V. Chernov).

Compiled by L.V. Enushevskaya

Translated by S. Belov 